\title{
Realistic Exact Solution for the Exterior Field of a Rotating Neutron Star
}

\author{
Leonardo. A. Pachón \\ Escuela de Física, Universidad Industrial de Santander, A.A. 678, Bucaramanga, Colombia and \\ Departamento de Física, Universidad Nacional, Bogotá D.C., Colombia \\ Jorge A. Ruedat \\ Escuela de Física, Universidad Industrial de Santander, A.A. 678, Bucaramanga, Colombia and \\ Centro de Física Teórica, Universidad de Los Andes, Mérida, Mérida 5101, Venezuela \\ José D. Sanabria-Gómet团 \\ Escuela de Física, Universidad Industrial de Santander, A.A. 678, Bucaramanga, Colombia
}

(Dated: September 6, 2018)

\begin{abstract}
A new six-parametric, axisymmetric and asymptotically flat exact solution of Einstein-Maxwell field equations having reflection symmetry is presented. It has arbitrary physical parameters of mass, angular momentum, mass-quadrupole moment, current octupole moment, electric charge and magnetic dipole, so it can represent the exterior field of a rotating, deformed, magnetized and charged object; some properties of the closed-form analytic solution such as its multipolar structure, electromagnetic fields and singularities are also presented. In the vacuum case, this analytic solution is matched to some numerical interior solutions representing neutron stars, calculated by Berti \& Stergioulas [1], imposing that the multipole moments be the same. As an independent test of accuracy of the solution to describe exterior fields of neutron stars, we present an extensive comparison of the radii of innermost stable circular orbits (ISCOs) obtained from Berti \& Stergioulas numerical solutions, Kerr solution [2], Hartle \& Thorne solution [3], an analytic series expansion derived by Shibata \& Sasaki [4] and, our exact solution. We found that radii of ISCOs from our solution fits better than others with realistic numerical interior solutions.
\end{abstract}

PACS numbers: 04.20.Jb, 04.40.Nr, 95.30.Sf, 98.80.Jk, 02.30.Em

\section{INTRODUCTION}

Observed pulsars are assumed to be highly deformed objects due to rotation. The spin frequencies of the 11 nuclear-powered pulsars lie between $230 \mathrm{~Hz}$ (e.g. for the PSR 1845-19 pulsar) and 641-Hz (e.g. for the PSR B1937+21 pulsar [5], 6]), and within that range are marginally consistent with a uniform distribution (see [7], 8] and references therein). The fast rotation is very important, not only in newly-born neutron stars which may undergo secular and dynamical instabilities (see 9 and references therein) but also in strange stars.

Studying Neutron Stars (NS) is interesting for several reasons. The quasi-stationary evolution of an isolated NS can be tracked considering equilibrium sequences in which the rest mass is constant while the angular momentum varies 10]. Such evolution may be driven by the adiabatic loss of energy and angular momentum via electromagnetic or gravitational radiation. On the other hand, the axisymmetric pulsations of rotating NS can be

\footnotetext{
*Electronic address: lpachon@laft.org

†Electronic address: jrueda@ula.ve

$\ddagger$ Electronic address: jsanabri@uis.edu.co
}

excited in several scenarios, like core collapse, crust - and core - quakes or binary mergers, and could become detectable in either gravitational waves or high-energy radiation [11]. Furthermore, the observational detection of such pulsations will yield valuable information about the equation of state of relativistic stars and therefore information about the properties and behavior of the matter at extreme conditions of high density $\left(\sim 10^{15} \mathrm{gr} / \mathrm{cm}^{3}\right)$ and temperatures (around $10^{6} \mathrm{~K}$ ) [12]. In addition, new aspects of rotating NS have been revealed in about 1000 pulsars. Some X-ray pulsars and some $\gamma$-ray pulsars have been detected in the past years. Among these new objects, some exhibit quite different behaviour in their pulse periods [13. The measurement of the period and its time derivative yields evidence of ultra-magnetized stars, possibly representing magnetars $[14$.

In the last two decades, great advances have been made to understand the properties of astrophysical objects like White Dwarfs, Black Holes or NSs, in the frame of General Relativity. Particularly, from the numerical approach, several physical properties of NS such as energy density, inertia moments, equatorial radii and others are known through the numerical solution of the full Einstein equations, assuming different equations of state for NS and applying algorithms, specially the KomatsuEriguchi-Hashisu self consistent field-method [15] and its variants (e.g. in 2005 by Ghezzi 16], Gusakov, Yakovlev 
\& Gnedin 17 and Berti, White, Maniopoulou \& Bruni 9]. In 2004 by Berti \& Stergioulas 1] and Yoshida \& Eriguchi 18]. In previous years by Stute \& Camenzind in 2003 [19], Manko, Mielke \& Sanabria-Gómez in 2000 20], Sibgatullin \& Sunyaev [21] in 1998, Bocquet Bonazzola, Gourgoulhon \& Novak 22], in 1995, and by Cook, Shapiro \& Teukolsky in 1994 [10] and 1992 [23]. and references therein). On the other hand, from the analytic close approach, to construct axisymmetric stationary exact solutions of the Einstein-Maxwell equations with physical parameters defined from the beginning is not a problem now, because of the development of the powerful integral method by Sibgatullin (see 24 and 25]), which allows to do this by considering the choosing of the Ernst's potentials on the symmetry axis.

The aim of this paper is to present a new stationary axially symmetric exact solution to the Einstein-Maxwell system as a model for the exterior field of a NS, not only for rapidly but also for slowly rotation rates. A consistent analytic closed representation of the exterior spacetime around a rapidly rotating NS is desirable for many reasons, among them: (i) if we have an analytic closed form for the metric, the computation of the stationary properties of the space-time is less difficult (for example, geodesics in the exterior of NS could be studied analytically 27] 26]; one could also find closed form expressions for the radii and frequencies of the innermost stable circular orbits (ISCOs) 27] [4], etc.), (ii) it could be useful for studying the dynamical properties of the space-time, like gravitational wave emission and chaotic trajectories of particles around it. Furthermore, having an analytic solution could simplify the calculation of properties of accretion disks, epicyclic frequencies [21] and so on.

Some analytic models for NS have been constructed and studied. Since the seminal works of Hartle [28] and Hartle-Thorne [3], the analytic structure of the spacetime outside a slowly rotating NS was generally associated with the Kerr solution because of its simplicity and accuracy with numerical data obtained from numerical interior solutions. Nevertheless, Kerr solution does not present an accurate fit with the expected values of some of the physical properties of realistic stars in the regime of rapid rotation rates (like the radii of ISCOs, see [1, 10, 19] and the mass-quadrupole moment [29]). Sibgatullin \& Sunyaev [21] adjusted the free parameters of the exact solution by Manko, Martin, Ruíz, Sibgatullin \& Zaripov in 30. with the numerical data for the ISCO and the gravitational redshift of Cook, Shapiro \& Teukolsky [10]. They analyzed the effects of the mass-quadrupole moment of a rapidly rotating NS on the energy release in the equatorial layer on the surface of the accreting star and in the accretion disk, using the normal sequences of Equations of State (EOS) A [31], AU [32] and FPS [33].

In 2000, Manko, Mielke \& Sanabria-Gómez [20] derived the charged, magnetized generalization with arbitrary quadripolar deformation of the Tomimatsu-Sato $\delta=2$ solution [34] written in rational functions, and claimed this solution as a possible model to describe the exterior field of a rotating NS. Then, Stute \& Camenzind [19] decided to study that solution to describe the gravitational field of a rapidly rotating NS. They matched the solution by Manko et al. with the numerical interior solutions from Cook et al 10 by a similar procedure to the one used in [21], concluding that the accuracy of that solution is high in the case of the redshift, but poor describing the radius of the marginally stable orbit (the matching was made using non invariants local properties of the solution, as it was pointed out in [1]). However, they considered that Manko et al. solution was a great improvement compared with Hartle \& Thorne solution in the regime of fast rotation.

Recently, the full Einstein equations were solved in a numerical approach by Berti \& Stergioulas (B\&S) [1], to determine the NS space-time along of sequences of constant rest mass for selected EOS (denoted as EOS A 31], EOS AU 32], EOS FPS 33], EOS L 35] and EOS APRb [36]). They matched the Manko et. al. solution [20] to the numerical solutions imposing the condition that the mass-quadrupole moment of the numerical and analytic space-times be the same, concluding that the matching condition can be satisfied only for very rapidly rotating stars. This affirmation was based on the fact that this exact solution does not reduce continuously to the Schwarzschild one when the rotation vanishes and, according to [1], the mass-quadrupole moment in the nonrotating limit (angular moment equals to zero) is very large for this limit, i.e., $Q=-m\left(m^{2}+b^{2}\right)^{2} /\left(4\left(m^{2}-b^{2}\right)\right)$, where $m$ is the mass and $b$ is a parameter related with the arbitrary mass-quadrupole moment respectively. For that reason, it is not possible that the solution by Manko et al. can describe NSs in the regime of slow rotations. Besides, B\&S suggested that for intermediate rotation rates could be used the exterior approximated analytic solution by Hartle \& Thorne [3], valid to second order in the rotation rate.

Following their own suggestion, in 2005 Berti et al. 9 compared the Hartle-Thorne [3] slow-rotation approximation keeping terms up to second order in the stellar angular velocity, and Manko et al. solution [20] again with their numerical solutions to the full Einstein's equations. Using the same matching procedure presented in [1], they found that the Hartle-Thorne approximation offers very good predictions for the corotating $R_{+}$and counterrotantig $R_{-}$ISCO radii, with $R_{ \pm}$accurate better than $1 \%$ even for the fastest millisecond pulsars. At these rotational rates the accuracy on the mass-quadrupole moment is $\sim 20 \%$, and better for longer periods.

In spite of the fact that the rich phenomenology ob- 
served from pulsars motivates the study of the interior and exterior electromagnetic fields of rotating NS, we use the same procedure exposed in [1], i.e., the matching of the multipole moments in the vacuum case, because of the limited information available about electromagnetic properties in numerical interior solutions to EinsteinMaxwell system. However, considering that the study of electromagnetic fields in strongly curved space-times has been the subject of past and recent interest, we also present the closed form and some properties of the electromagnetic field of our solution.

This paper is presented in the following order. In section 2, we present the six-parametric solution including issues about its construction. In section 3, we summarize the properties of this solution through its multipolar structure, limiting cases, electromagnetic fields and singularities. In section 4, we match our analytic solution with the interior numerical solutions by Berti \& Stergioulas (hereafter $\mathrm{B} \& \mathrm{~S}$ ) and present comparisons of the radii of ISCOs of several numerical and exact solutions. Finally, concluding remarks about the paper are written.

\section{SIX-PARAMETER SOLUTION}

In order to construct a realistic exact solution to the Einstein-Maxwell equations, we must consider some physical assumptions. In Newtonian Theory of Gravitation all equilibrium states of isolated self-gravitating fluids (i.e. stellar models) must have reflection symmetry through a plane which is perpendicular to the rotation axis of the star [37]; according to that idea, it has been conjectured that stationary general relativistic stellar models must have reflection symmetry as well [38]. For the stationary axisymmetric case a simple form of the metric was given by Papapetrou [39], that is

$$
d s^{2}=-f(d t-\omega d \phi)^{2}+f^{-1}\left[e^{2 \gamma}\left(d \rho^{2}+d z^{2}\right)+\rho^{2} d \phi^{2}\right],
$$

where $f, \omega$ and $\gamma$ are functions of the quasi-cylindrical Weyl coordinates $(\rho, z)$.

Using the above line element, the Einstein-Maxwell equations can be reformulated, via Ernst's procedure [40], in terms of two complex potentials $\mathcal{E}$ and $\Phi$ as follow:

$$
\begin{aligned}
\left(\operatorname{Re} \mathcal{E}+|\Phi|^{2}\right) \nabla^{2} \mathcal{E} & =\left(\nabla \mathcal{E}+2 \Phi^{*} \nabla \Phi\right) \cdot \nabla \mathcal{E} \\
\left(\operatorname{Re} \mathcal{E}+|\Phi|^{2}\right) \nabla^{2} \Phi & =\left(\nabla \mathcal{E}+2 \Phi^{*} \nabla \Phi\right) \cdot \nabla \Phi
\end{aligned}
$$

In this paper, we solve the Ernst's equations (2) with the aid of the Sibgatullin's method [25] 24], according to which the complex potentials $\mathcal{E}$ and $\Phi$ can be calculated from specified axis data $e(z):=\mathcal{E}(z, \rho=0)$ and
$f(z):=\Phi(z, \rho=0)$, by the integrals

$$
\begin{aligned}
& \mathcal{E}(z, \rho)=\frac{1}{\pi} \int_{-1}^{1} \frac{e(\xi) \mu(\sigma) d \sigma}{\sqrt{1-\sigma^{2}}} \\
& \Phi(z, \rho)=\frac{1}{\pi} \int_{-1}^{1} \frac{f(\xi) \mu(\sigma) d \sigma}{\sqrt{1-\sigma^{2}}}
\end{aligned}
$$

The unknown function $\mu(\sigma)$ must satisfy the singular integral equation

$$
f_{-1}^{1} \frac{\mu(\sigma)[e(\xi)+\tilde{e}(\eta)+2 f(\xi) \tilde{f}(\eta)] d \sigma}{(\sigma-\tau) \sqrt{1-\sigma^{2}}}=0
$$

and the normalizing condition

$$
\int_{-1}^{1} \frac{\mu(\sigma) d \sigma}{\sqrt{1-\sigma^{2}}}=\pi
$$

where $\xi=z+i \rho \sigma, \eta=z+i \rho \tau, \rho$ and $z$ being the WeylPapapetrou quasi-cylindrical coordinates, $\sigma, \tau \in[-1,1]$, $\tilde{e}(\eta):=\overline{e(\bar{\eta})}, \tilde{f}(\eta):=\overline{f(\bar{\eta})}$ and the overbar stands for complex conjugation.

With the purpose to construct reflectionally symmetric solutions, the Ernst's potentials on the symmetry axis $e(z)$ and $f(z)$ must satisfy the following conditions

$$
e(z) \bar{e}(-z)=1 \quad \text { and } \quad f(z)=-\bar{f}(-z) e(z),
$$

when the electromagnetic potentials are even functions of $z$ [41]. One choice of Ernst's potentials satisfying the above considerations is [42]

$$
\begin{aligned}
& e(z)=\frac{z^{3}-z^{2}(m+i a)-k z+i s}{z^{3}+z^{2}(m-i a)-k z+i s}, \\
& f(z)=\frac{q z^{2}+i \mu z}{z^{3}+z^{2}(m-i a)-k z+i s},
\end{aligned}
$$

where $m$ denotes the gravitational mass of the source, $a$ its specific angular momentum, $q$ its electric charge, and $k, s$ and $\mu$ parameters related with the mass-quadrupole moment, the current octupole and the dipolar magnetic moment respectively (see Subsection $\amalg$ IIA). We have included the parameter $s$ following the suggestion given in [19] regarding to the Manko et al. solution, whose accuracy is not good for the radius of the marginallystable-orbit, which is more sensitive to higher multipole moments of the space-time. It seems to show that higher multipole moments ought to be included, especially for rapid rotation and stiffer equation of state, to improve the freedom to fit the exterior gravitational field of neutron stars with numerical interior solutions. Moreover, this parameter could aid us to understand the influence of the differential rotation in the dynamics properties of the surrounding space-time. 
The potentials (7) can be written in an alternative way, we mean

$$
e(z)=1+\sum_{i=3}^{3} \frac{e_{i}}{z-\beta_{i}}, \quad f(z)=\sum_{i=3}^{3} \frac{f_{i}}{z-\beta_{i}}
$$

with

$$
\begin{array}{r}
e_{j}=(-1)^{j} \frac{2 m \beta_{j}^{2}}{\left(\beta_{j}-\beta_{k}\right)\left(\beta_{j}-\beta_{i}\right)}, \\
f_{j}=(-1)^{j+1} \frac{i \mu \beta_{j}+q \beta_{j}^{2}}{\left(\beta_{j}-\beta_{k}\right)\left(\beta_{j}-\beta_{i}\right)}, \quad i, k \neq j .
\end{array}
$$

Then, using (2) and (7), following a similar procedure to the one used in [44], we obtain the Ernst potentials and the metric functions in whole space-time:

$$
\begin{gathered}
\mathcal{E}=\frac{A+B}{A-B}, \quad \Phi=\frac{C}{A-B}, \\
f=\frac{A \bar{A}-B \bar{B}+C \bar{C}}{(A-B)(\bar{A}-\bar{B})}, \quad e^{2 \gamma}=\frac{A \bar{A}-B \bar{B}+C \bar{C}}{K \bar{K} \prod_{n=1}^{6} r_{n}}, \\
\omega=\frac{\operatorname{Im}[(A+B) \bar{H}-(\bar{A}+\bar{B}) G-C \bar{I}]}{A \bar{A}-B \bar{B}+C \bar{C}},
\end{gathered}
$$

where

$$
\begin{aligned}
A & =\sum_{1 \leq i<j<k \leq 6} a_{i j k} r_{i} r_{j} r_{k}, \quad B=\sum_{1 \leq i<j \leq 6} b_{i j} r_{i} r_{j} \\
C & =\sum_{1 \leq i<j \leq 6} c_{i j} r_{i} r_{j}, \quad H=z A-\left(\beta_{1}+\beta_{2}+\beta_{3}\right) B \\
& +\sum_{1 \leq i<j<k \leq 6} h_{i j k} r_{i} r_{j} r_{k}+\sum_{1 \leq i<j \leq 6}\left(\alpha_{i}+\alpha_{j}\right) b_{i j} r_{i} r_{j} \\
G & =-\left(\beta_{1}+\beta_{2}+\beta_{3}\right) A+z B+\sum_{1 \leq i<j \leq 6} g_{i j} r_{i} r_{j} \\
& +\sum_{1 \leq i<j<k \leq 6}\left(\alpha_{i}+\alpha_{j}+\alpha_{k}\right) a_{i j k} r_{i} r_{j} r_{k}, \\
I & =\left(f_{1}+f_{2}+f_{3}\right)(A-B)+\left(\beta_{1}+\beta_{2}+\beta_{3}-z\right) C \\
& +\sum_{1 \leq i<j<k \leq 6} p_{i j k} r_{i} r_{j} r_{k}+\sum_{i=1} p_{i} r_{i} \\
& +\sum_{1 \leq i<j \leq 6}\left[p_{i j}-\left(\alpha_{i}+\alpha_{j}\right) c_{i j}\right] r_{i} r_{j}
\end{aligned}
$$

with

$$
\begin{aligned}
r_{i} & =\sqrt{\rho^{2}+\left(z-\alpha_{i}\right)^{2}} \\
a_{i j k} & =(-1)^{i+j+1} \Lambda_{i j k} \Gamma_{l \mid m n}, \\
b_{i j} & =(-1)^{i+j} \lambda_{i j} H_{l \mid m n p}, \\
c_{i j} & =(-1)^{i+j} \lambda_{i j}\left[f\left(\alpha_{l}\right) \Gamma_{m \mid n p}-f\left(\alpha_{m}\right) \Gamma_{n \mid p l}\right. \\
& \left.+f\left(\alpha_{n}\right) \Gamma_{p \mid l m}-f\left(\alpha_{p}\right) \Gamma_{l \mid m n}\right] \\
h_{i j k} & =(-1)^{i+j+k} \Lambda_{i j k}\left(e_{1}^{*} \delta_{23 \mid l m n}+e_{2}^{*} \delta_{31 \mid l m n}\right. \\
& \left.+e_{3}^{*} \delta_{12 \mid l m n}\right) \\
g_{i j} & =(-1)^{i+j} \lambda_{i j}\left(\alpha_{l} \Gamma_{m \mid n p}-\alpha_{m} \Gamma_{n \mid p l}\right. \\
& \left.+\alpha_{n} \Gamma_{p \mid l m}-\alpha_{p} \Gamma_{l \mid m n}\right), \\
p_{i} & =(-1)^{i} D_{i}\left[f\left(\alpha_{l}\right) H_{m \mid n p s}-f\left(\alpha_{m}\right) H_{n \mid p s l}\right. \\
& +f\left(\alpha_{n}\right) H_{p \mid s l m}-f\left(\alpha_{p}\right) H_{s \mid l m n} \\
& \left.+f\left(\alpha_{s}\right) H_{l \mid m n p}\right], \\
p_{i j} & =(-1)^{i+j} \lambda_{i j}\left(e_{1}^{*} \Upsilon_{23 \mid l m n p}+e_{2}^{*} \Upsilon_{31 \mid l m n p}\right. \\
& \left.+e_{3}^{*} \Upsilon_{12 \mid l m n p}\right) \\
p_{i j k} & =(-1)^{i+j+1} \Lambda_{i j k}\left(e_{1}^{*} \Psi_{23 \mid l m n}+e_{2}^{*} \Psi_{31 \mid l m n}\right. \\
& \left.+e_{3}^{*} \Psi_{12 \mid l m n}\right)
\end{aligned}
$$

and

$$
\begin{aligned}
\lambda_{i j} & =\left(\alpha_{i}-\alpha_{j}\right) D_{i} D_{j}, \\
\Lambda_{i j k} & =\left(\alpha_{i}-\alpha_{j}\right)\left(\alpha_{i}-\alpha_{k}\right)\left(\alpha_{j}-\alpha_{k}\right) D_{i} D_{j} D_{k}, \\
D_{i} & =\frac{1}{\left(\alpha_{i}-\beta_{1}\right)\left(\alpha_{i}-\beta_{2}\right)\left(\alpha_{i}-\beta_{3}\right)}, \\
\Gamma_{l \mid m n}= & H_{3}\left(\alpha_{l}\right) \Delta_{12 \mid m n}+H_{3}\left(\alpha_{m}\right) \Delta_{12 \mid n l} \\
+ & H_{3}\left(\alpha_{n}\right) \Delta_{12 \mid l m}, \\
\Delta_{l m \mid n p}= & H_{l}\left(\alpha_{n}\right) H_{m}\left(\alpha_{p}\right)-H_{l}\left(\alpha_{p}\right) H_{m}\left(\alpha_{n}\right), \\
H_{l}\left(\alpha_{n}\right)= & \frac{2 \prod_{p \neq n}\left(\alpha_{p}-\beta_{l}^{*}\right)}{\prod_{k \neq l}^{3}\left(\beta_{l}^{*}-\beta_{k}^{*}\right) \prod_{k=1}^{3}\left(\beta_{l}^{*}-\beta_{k}\right)} \\
- & 2 \sum_{k=1}^{3} \frac{f_{l}^{*} f_{k}}{\left(\beta_{l}^{*}-\beta_{k}\right)\left(\alpha_{n}-\beta_{k}\right)}, \\
\delta_{l m \mid n p s}= & \Delta_{l m \mid n p}+\Delta_{l m \mid p s}+\Delta_{l m \mid s n}, \\
h_{l \mid m n p}= & H_{3}\left(\alpha_{l}\right) \delta_{12 \mid m n p}, \\
H_{l \mid m n p}= & h_{l \mid m n p}+h_{m \mid n p l}+h_{n \mid p l m}+h_{p \mid l m n}, \\
\Psi_{l m \mid n p s}= & f\left(\alpha_{n}\right) \Delta_{l m \mid p s}+f\left(\alpha_{p}\right) \Delta_{l m \mid s n}+f\left(\alpha_{s}\right) \Delta_{l m \mid n p}, \\
\Upsilon_{l m \mid n p r s}= & f\left(\alpha_{n}\right) \delta_{l m \mid p r s}-f\left(\alpha_{p}\right) \delta_{l m \mid r s n} \\
+ & f\left(\alpha_{r}\right) \delta_{l m \mid s n p}-f\left(\alpha_{s}\right) \delta_{l m \mid n p r}, \\
\text { being } \alpha \text { 's the roots of the Sibgatullin's equation }[25] & e(z)+\tilde{e}(z)+2 \tilde{f}(z) f(z)=0 . \\
& \\
& \\
& \\
& \\
& \\
&
\end{aligned}
$$




\section{PROPERTIES OF THE SOLUTION}

\section{A. Multipolar structure}

This solution describes the exterior gravitational field of a reflectionally symmetric source. Their first four relativistic mass multipolar moments and first two electromagnetic multipolar moments are arbitrary. The multipolar moments are calculated using the Fodor-HoenselaersPerjés procedure 43] following the parametrization given in (7), which has the advantange that the real parameters $m$ corresponds to the total mass $M, a$ to the total angular moment per unit mass $(a=J / m$, being $J$ the total angular moment) and $q$ to the total electric charge $Q$; while $k, s$ and $\mu$ represent the mass-quadrupole moment $M_{2}$, current octupole $S_{3}$ and magnetic dipole $\mathcal{B}$ through the following formulas:

$$
\begin{aligned}
M_{2} & =m k-m a^{2} \\
S_{3} & =2 i m a k-i a m^{3}-i m s \\
\mathcal{B} & =i \mu+i a q .
\end{aligned}
$$

The solution also has electric charge quadrupole moment $\mathcal{Q}_{2}$, which is not independent because it depends on the other multipoles, given by the formula

$$
\mathcal{Q}_{2}=-a \mu-a^{2} q+k q,
$$

and permits that the solution has electric field even when $q$ vanish (induced electric field by rotation [45]).

For NSs the mass-quadrupole moment is, surprisingly, approximated by the simple quadratic relation [29]

$$
M_{2} \approx-c(M, E O S) \frac{J^{2}}{M},
$$

where the constant $c=c(M, E O S)$ depends only on the mass $\mathrm{M}$ and the equation of state (EOS) for the interior of the NS. So, the formulas (7) are successfull to fit $M_{2}$ of the exact solucion with data of numerical solutions because of the form of $M_{2}$ and the freedom to set the numerical value of the real parameter $k$. In addition, the absence of the gravitomagnetic parameter (monopole of angular momentum) leads to achieve the asymptotic flatness condition of the solution, necessary to describe a compact object.

\section{B. Limiting Cases}

A special feature of this solution is that in rigid rotation, i.e., with $s=0$, it contains the following well-known limiting cases:
1. The stationary vacuum case $q=0, \mu=0$ with nonvanishing arbitrary deformation parameter $k=$ $-1 / 4\left(m^{2}-a^{2}\right)$ represents the Tomimatsu-Sato $\delta=$ 2 solution [34] with the mass- quadrupole moment $M_{2}=-1 / 4\left(m^{3}-J^{2} / m\right)$.

2. The magnetostatic limit $q=0, a=0$ represents the massive magnetic dipole solution of Bonnor [46].

3. The Kerr-Newmann space-time is obtained with $\mu=k=0$.

4. The stationary vacuum case $q=0, \mu=0$ with nonarbitrary deformation, i.e., with $k=0$ represents the Kerr space-time.

It is important to emphasize that the present six parametric solution generalizes the Kerr and Kerr-Newmann solutions, which are the most studied stationary axisymmetric solutions.

\section{Electromagnetic Fields}

The strong magnetic field is one of the most important features of NSs and pulsars. The last ones have nonaligned dipole moment with the rotation axis causing loss of energy and angular moment, so the star radiates electromagnetic and gravitational waves away and decrease its velocity of rotation, configuring a clear example of a nonstationary and nonaxisymmetric system. On the other hand, the stationary axisymmetric case is characterized by the fact that the magnetic dipole moment is aligned with the axis rotation of the star and the magnetic field is poloidal (i.e. the magnetic field lying in the meridional planes 22]). Our exact solution is stationary and axisymmetric and, therefore, describes this kind of NSs.

For the description of the electromagnetic properties of the solution, we must to calculate the electric and magnetic fields produced in the surrounding space-time. The reason to introduce electric field in the solution, in spite of the fact of global neutrality of the astrophysical objects, is that an electric field is induced by the rotation of the magnetized objects, as Eq. (11) shows. To have a closed form the electric and magnetic fields is important, for example, in order to study the accretion of charged particles around of the NS, to study geodesic motion of test particles around a NS and so on. The electric and magnetic fields can be calculated using the expressions

$$
E_{\alpha}=F_{\alpha \beta} u^{\beta}, \quad B_{\alpha}=-\frac{1}{2} \epsilon_{\alpha \beta}^{\gamma \delta} F_{\gamma \delta} u^{\beta},
$$

where $F_{\alpha \beta}$ is the electromagnetic field tensor $F_{\alpha \beta}=$ $2 A_{[\beta ; \alpha]}, A_{\mu}=\left(0,0, A_{\phi},-A_{t}\right)$ is the electromagnetic fourpotential, $\epsilon_{\alpha \beta \gamma \delta}$ is the totally antisymmetric tensor the 
positive orientation with norm $\epsilon_{\alpha \beta \gamma \delta} \epsilon^{\alpha \beta \gamma \delta}=-24$ [4] and $u_{\alpha}$ is a time-like vector (in the case of fluid, this vector is the four-velocity of the fluid). For a congruence of observers at rest in the frame of (1), the four-velocity is defined by the time-like vector

$$
u^{\alpha}=(1 / \sqrt{f}, 0,0,0),
$$

so the vectorial fields have components in the $\rho$ and $\phi$ directions. The electric field is given by the following expressions:

$$
E_{\rho}=-\frac{\sqrt{f}}{e^{2 \gamma}} A_{t, \rho}, \quad E_{z}=-\frac{\sqrt{f}}{e^{2 \gamma}} A_{t, z},
$$

and the magnetic field by

$$
\begin{aligned}
& B_{\rho}=\frac{f^{3 / 2}}{\rho e^{2 \gamma}}\left(-\omega A_{t, z}+A_{\phi, z}\right), \\
& B_{z}=-\frac{f^{3 / 2}}{\rho e^{2 \gamma}}\left(-\omega A_{t, \rho}+A_{\phi, \rho}\right) .
\end{aligned}
$$

The $A_{t}$ potential is the real part of the electromagnetic Ernst potential $\Phi$ given by (8), and the potential $A_{\phi}$ can be calculated as the real part of the Kinnersley potential $\mathcal{K}=A_{\phi}+i A_{t}^{\prime}$ [8], which can be obtained using the Sibgatullin method and can be written as

$$
\mathcal{K}=-i \frac{I\left(f_{1}+f_{2}\right)}{A-B}
$$

Thus, the closed-form expressions for the electric and magnetic fields are

$$
\begin{gathered}
E_{\rho}=-\frac{|K|^{2} \prod_{n=1}^{6} r_{n}}{|A-B| \sqrt{|A|^{2}-|B|^{2}+|C|^{2}}} \operatorname{Re}\left\{\left[\frac{C_{, \rho}-C \ln (A-B)_{, \rho}}{A-B}\right]\right\} \\
E_{z}=-\frac{|K|^{2} \prod_{n=1}^{6} r_{n}}{|A-B| \sqrt{|A|^{2}-|B|^{2}+|C|^{2}}} \operatorname{Re}\left\{\left[\frac{C_{, z}-C \ln (A-B)_{, z}}{A-B}\right]\right\} \\
B_{\rho}=\frac{\operatorname{Im}(A-B) \bar{H}+(\bar{A}-\bar{B}) G-C \bar{I}]}{\rho|A-B|^{2}} E_{z}+\frac{|K|^{2} \sqrt{|A|^{2}-|B|^{2}+|C|^{2}} \prod_{n=1}^{6} r_{n}}{\rho|A-B|^{3}} \operatorname{Im}\left\{\frac{\left(\bar{f}_{1}+\bar{f}_{2}\right)\left[\bar{I}_{, z}-\bar{I} \ln (\bar{A}-\bar{B})_{, z}\right]}{A-B}\right\} \\
B_{z}=-\frac{\operatorname{Im}(A-B) \bar{H}+(\bar{A}-\bar{B}) G-C \bar{I}]}{\rho|A-B|^{2}} E_{\rho}+\frac{|K|^{2} \sqrt{|A|^{2}-|B|^{2}+|C|^{2}} \prod_{n=1}^{6} r_{n}}{\rho|A-B|^{3}} \operatorname{Im}\left\{\frac{\left(\bar{f}_{1}+\bar{f}_{2}\right)\left[\bar{I}_{, \rho}-\bar{I} \ln (\bar{A}-\bar{B})_{, \rho}\right]}{A-B}\right\} .
\end{gathered}
$$

In order to show that our solution have the appropriate features of realistic NSs, we have to use typical values of NS for their physical parameters, like mass, rotation, deformation, charge and magnetic dipole moment. NS are compact objects that possess strong magnetic fields $\left(B \sim 10^{12} T\right)$, nevertheless, in our solution the parameter related with the magnetic field is the magnetic dipole moment $\mu$. In [20] Manko et. al. showed how the magnetic dipole moment of their exterior solution depends on the values of the parameters of the interior solution, such as the matter density, the current function and the stellar radius. They obtained the above result by comparison between their magnetic potential $A_{\phi}$ at infinite and the Ferraro's potential [49]; this became a test for the weak field because the Ferraro's solution is an approximate one that have into account deformation only at first order around the spherical symmetry 22]. According to this, we must to use typical values given for realistic interior solutions. Bocquet et al. 22] calculated interior solutions for the Einstein-Maxwell system using the typical value of $10^{32} \mathrm{~A} \mathrm{~m}^{2}$ for the magnetic dipolar moment of the NS in S.I. units. In natural units $\left(c=G=\mu_{0}=\varepsilon_{0}=1\right)$, which have been used in our paper, the magnetic dipole moment has a order of

$$
\mu_{\text {natural }} \sim \frac{10^{-6} \sqrt{\mu_{0} G}}{c^{2}} \mu_{\text {S.I. }},
$$

resulting a typical value for the $\mu$ parameter of our solution of order $10^{1} \mathrm{Km}^{2}$. 
In the Fig. 11 we have plotted the force lines of the magnetic field and the isopotential lines of the induced electric field ( $A_{t}=$ constant) for one possible model of NS $\left(M_{\odot}=1.47663 \mathrm{Km}\right)$. The increasing of the separation between consecutive force lines indicates, as is usual, that the electric field and the magnetic one decreases while the distance increases. As we expected, in absence of monopole electric charge distribution, the electric field is quadrupolar. In addition, the same figure shows that the axistationary properties of the magnetic field appear: aligned magnetic dipole moment and dipolar structure (poloidal field).

\section{Singularities}

The singularities are solutions of the equation

$$
A-B=0
$$

i.e., denominator of the Ernst potentials given by (8) equals to zero. In the Fig. 2 we have plotted the typical shapes of stationary limit surfaces $(f=0)$ for specific sets of realistic parameters given in Ref. [1]. The location of ring singularity appears with dots. The ring singularity lie on the stationary limit surface, which is a characteristic of the stationary vacuum solutions.

For all stellar models there are no ring singularities outside of infinite red shift surfaces, therefore the solution can represent the exterior field of compact objects. In addition, we show in this section, with an approximate calculation via Komar masses, that the ring singularities of solution are massless. The geometrical structure of the exact solution implies that one could consider it as superposition of subextreme and hyperextreme objects. The Komar mass $M_{i}$ of a subextreme part can be calculated using the Tomimatsu formula [50]

$$
M_{i}=\frac{1}{4} \int_{\alpha_{<}}^{\alpha_{>}} \omega^{(i)} \Omega_{, z} d z,
$$

where $\Omega$ is the imaginary part of the Ernst potential $\varepsilon$, and $\omega^{i}$ is the constant value of the metric coefficient $\omega$ on the segment $\left[\alpha_{<}, \alpha_{>}\right]$of the $z$-axis.

In the case of hyperextreme part, the Komar mass cannot be evaluated using the formula (23), and one more general integral expression should be used [51]

$$
\begin{array}{r}
M_{i}=\frac{1}{4}\left\{\int_{z_{l}}^{z_{u}}[\rho(\ln f), \rho-\omega \Omega, z]_{\rho=\rho_{0}} d z+\int_{0}^{\rho_{0}}[\rho(\ln f), z\right. \\
\left.\quad-\omega \Omega, \rho]_{z=z_{u}} d \rho-\int_{0}^{\rho_{0}}\left[\rho(\ln f),,_{z}-\omega \Omega, \rho\right]_{z=z_{l}} d \rho\right\}(2
\end{array}
$$

(a)

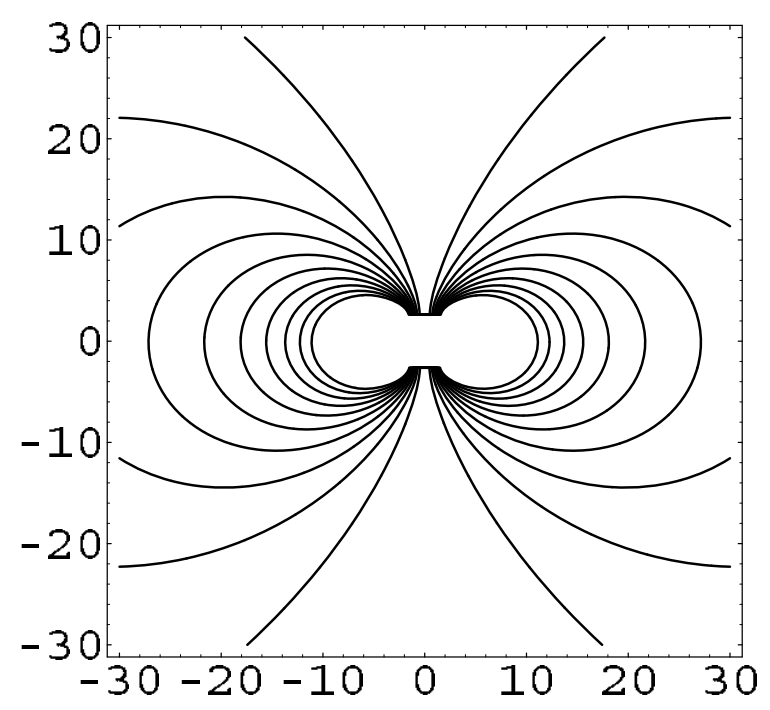

(b)

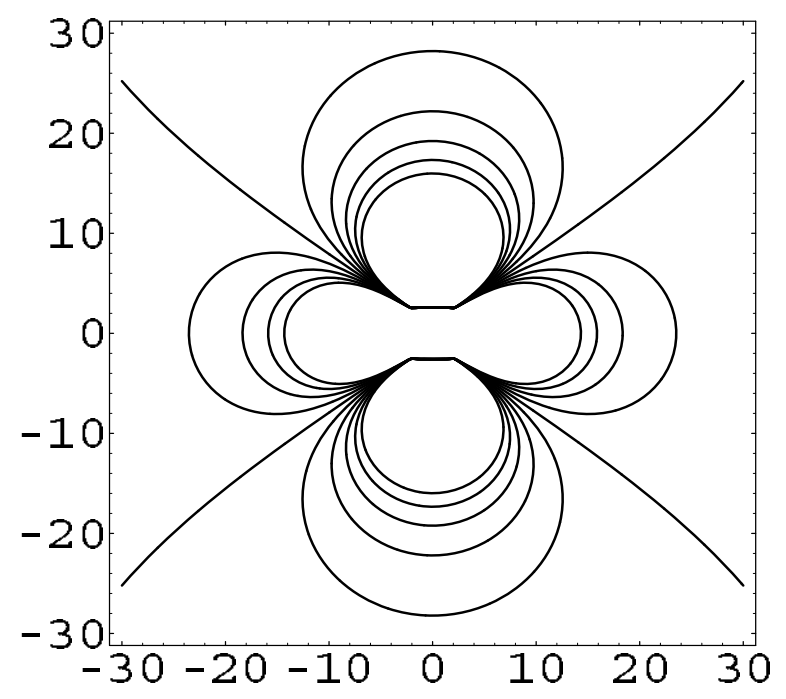

FIG. 1: (a) Magnetic field force lines and (b) isopotential lines of the induced electric field for $m=1.742 M_{\odot}, a=1.009 \mathrm{Km}$, $k=-0.336 \mathrm{Km}^{2}, s=0.212 \mathrm{Km}^{3}, q=0 \mathrm{Km}, b=10 \mathrm{Km}^{2}$.

where the integration is carried out over the surface of a cylinder enclosing the cut which joins the points $\alpha_{i}$ and $\bar{\alpha}_{i} ; z_{u}$ and $z_{l}$ denote locations on the symmetry axis of the centers of upper and lower bases, $\rho_{0}$ is the radius of the bases. We will illustrate the procedure followed using the set of parameters showed in the Fig. 2] First, we shall calculate the mass of the NS via Komar masses and then we will compare the obtained result with the mass monopole $m$, to deduce that the mass of the ring 


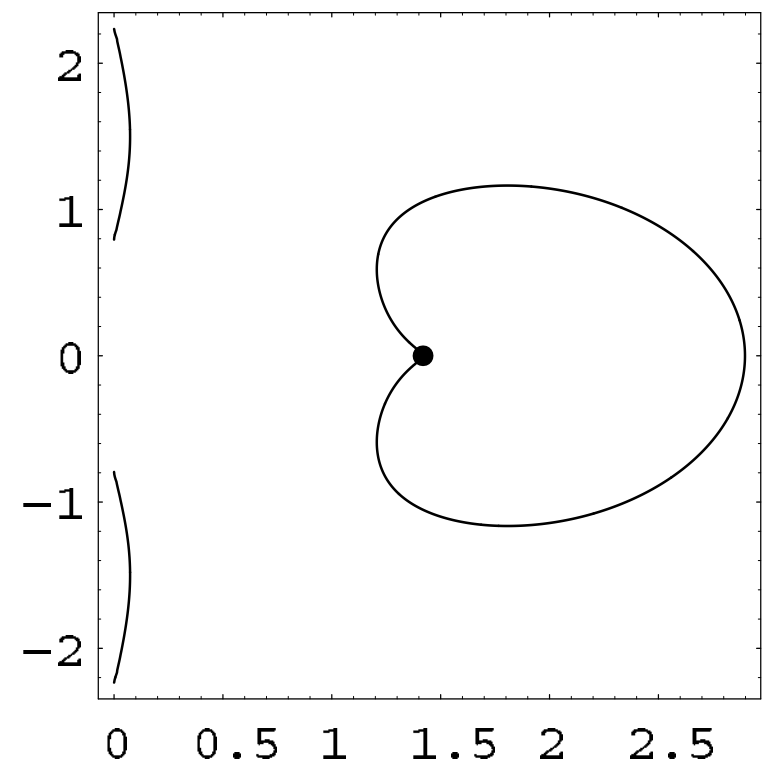

FIG. 2: Stationary limit surfaces for $m=2.388 M_{\odot}=$ $3.526 K m, a=2.264 K m, k=-1.808 K^{2} m^{2}, s=2.498 K m^{3}$.

singularity is equal to zero. For the set of parameters showed in Fig. 2 the roots of the Sibgatullin equation (9) are $\alpha_{1}=0.792, \alpha_{2}=2.245, \alpha_{3}=-0.792, \alpha_{4}=$ $-2.245, \alpha_{5}=-1.406 i, \alpha_{6}=\bar{\alpha}_{5}$, producing four subextreme parts and one hyperextreme part. Using the formula (23), the mass of the subextreme contribution is $m_{s}=1.523 \mathrm{Km}$ and using the formula (24), the mass of the hyperextreme contribution is $m_{h}=2.003 \mathrm{Km}$, then $m_{s}+m_{h}=3.526 \mathrm{Km}=m$. We made the above calculations for all data for all EOS used from Ref. [1] and the results were allways the same.

As can be seen, the calculations were made in a numerical way for the different stellar modells; it brings us to give only approximate values for the mass of ring singularity. For that reason, the ring singularity arise with a mass equals to zero with precision up to $10^{-4}$, with no dependence of the equation of state used.

\section{TEST OF ACCURACY OF THE SIX-PARAMETER ANALYTIC MODEL}

In order to demonstrate that our solution can represent the exterior gravitational field of a realistic NS, we have to do comparisons with results obtained from numerical interior solutions and with others possible analytic models. We have chosen the Kerr solution, the expres- sions derived by Shibata \& Sasaki (S\&S) in Ref. [4] and Hartle-Thorne's solution (H\&T) truncated to second order of the expansion parameter $\epsilon=\Omega / \Omega *$ (see Ref. 27] and $[9]$ ). For the comparison with numerical models, we have chosen the solutions by B\&S Ref. [1]; the comparison procedure was taken from there, that is, we compared an invariant quantity of the space-time, the multipolar moments, and other quantity which can be depending on the coordinates. The depending coordinates quantity could be the gravitational red shift or the radii of the Innermost Stable Circular Orbits (ISCOs). For this work we have chosen to compare the radii of the ISCO because it is related to several astrophysical properties of rapidly rotating NSs, e.g. the accretion disk cannot be longer that radii of the ISCO, and this sets an upper limit to the Keplerian frequency of particles orbiting a star.

We matched the exact solution with the numerical interior solutions imposing the condition that the first four mass and current multipole moments, i.e. mass, angular moment, mass-quadrupole and current octupole, of the exact solution and the numerical ones have the same value. In B\&S, sources possessing electric charge or magnetic dipolar moment were not considered, so in this work the comparison of the solutions was developed in the vacuum case.

The numerical solutions were calculated for five interior numerical solutions for the gravitational field of a NS, each numerical solution is described by a different Equation Of State (EOS). The EOS used were denoted as A 31], AU 32, FPS 33, L 35], APRb 36] (see B\&S [1] and Cook et al. [10] for details). These numerical solutions were calculated for three different sequences of constant rest mass:

- First sequence. The sequence corresponding to a canonical neutron star having gravitational mass $M=1.4 M_{\odot}$ in the non rotating limit.

- Second sequence. The sequence terminating at the maximum-mass model in the non rotating limit (maximum-mass normal sequence).

- Third sequence. A supramassive sequence, i.e., a sequence which does not terminate at a nonrotating model.

In order to distinguish when a neutron star is rapidly rotating or not, we shall use the dimensionless parameter $j$, defined by $j \equiv J / M^{2}$, as it was used by Sibgatullin \& Sunyaev [21], Stute \& Camenzind [19] and B\&S [1]. In spite of $j$ is not a direct quantitative measure of the magnitude of the rotation rate of the stars, it gives us a measure of the angular momentum which relates proportionally to the angular velocity. 
To clarify the meaning of the value of the $j$ parameter lets consider an example. For the recently discovered pulsar [6] which frequency is $716-\mathrm{Hz}$, the value of $j$ depends on the EOS and the sequence of mass considered. Using the values reported in $\mathrm{B} \& \mathrm{~S}$ for stars with similar frequency, we find that $j$ value must be around $0.42<j<0.68$. For example, for the EOS FPS in the first sequence of mass and assuming $m \sim 1.430 M_{\odot}$ and $J \sim 2.961 \mathrm{Km}^{2}$, the value of $j$ must be very close to 0.67 , and for the second sequence assuming $m \sim 1.831 M_{\odot}$ and $J \sim 3.185 \mathrm{Km}^{2}$, the value of $j$ must be very close to 0.43 . Although, it is clear that we can not present a reliable classification for rapidly rotating neutron stars based on $j$, we shall assume that a NS is considered rapidly rotating when $j>0.35$.

\section{A. Innermost stable circular orbits}

It is well known that not all orbits around relativistic stars are stable. For non-rotating stars, the ISCO is located at a circumferential radius of $R_{I S C O}=6 M$ (see Ref. 52] for a complete treatment of circular orbits in stationary axisymmetric spacetimes). Depending on the EOS and the mass of the star, the ISCO can be located outside the stellar surface. The rotation introduces a preferred direction in the $\phi$ coordinate, so ISCOs around a rotating star belong to two families: the co-rotating and the counter-rotating one.

A circular orbit in the equatorial plane is one for which $\rho=$ const. The equation for geodesic motion along the radial coordinate $\rho$ reads

$$
-g_{\rho \rho}\left(\frac{d \rho}{d \tau}\right)^{2}=1-\frac{E^{2} g_{\phi \phi}+2 E L g_{t \phi}+L^{2} g_{t t}}{g_{t \phi}^{2}-g_{t t} g_{\phi \phi}} \equiv V(\rho),
$$

where $E$ and $L$ are the conserved energy and angular momentum per unit mass, determined by the conditions $V=d V / d \rho=0$. Geodesics become marginally stable when $d^{2} V / d \rho^{2}=0$, so it is

$$
\begin{aligned}
& \omega^{\prime} \quad \omega^{\prime \prime} f^{5} \rho\left(2 f-f^{\prime} \rho\right)+\omega^{\prime 2} f^{4}\left[2 f^{2}+\left(-f^{\prime 2}+f^{\prime \prime} f\right) \rho^{2}\right] \\
& +\omega^{\prime} f^{2} \sqrt{\omega^{\prime 2} f^{4}+f^{\prime} \rho\left(2 f-f^{\prime} \rho\right)}\left[2 f^{2}+2 f^{\prime 2} \rho^{2}\right. \\
& -\quad f \rho\left(4 f^{\prime}+f^{\prime \prime} \rho\right)+\rho\left(2 f-f^{\prime} \rho\right)\left\{3 f^{\prime} f^{2}\right. \\
& -4 f^{\prime 2} f \rho+f^{\prime 3} \rho^{2}+f^{2}\left[f^{\prime \prime} \rho\right. \\
& \left.\left.-\quad \omega^{\prime \prime} f \sqrt{\omega^{\prime 2} f^{4}+f^{\prime} \rho\left(2 f-f^{\prime} \rho\right)}\right]\right\}=0
\end{aligned}
$$

for co-rotating orbits $\left(R_{+}\right)$, where ' indicates a partial derivative with respect to $\rho$. For the case of HartleThorne's solution, the radii of the ISCO for co-rotating orbits $\left(R_{+}\right)$was derived by Abramowicz et al. in [27]:

$$
\begin{aligned}
R_{I S C O}^{H \& T} & =6 m\left[1-\sqrt{\frac{8}{27}} j+\left(\frac{251647}{2592}-240 \ln \frac{3}{2}\right) j^{2}-\right. \\
& \left.-\left(\frac{9325}{96}-240 \ln \frac{3}{2}\right) q\right]
\end{aligned}
$$

where $q$ is a parameter related with the cuadripolar deformation and defined as $q=Q / \mathrm{m}^{3}$, where $Q$ is the cudripolar deformation multipole.

S\&S derived a general representation of axisymmetric vacuum solutions (in the form of a series expansion of the physical multipole moments of the space-time) and found some approximate analytic formulas for the location of the inner stable circular orbit, the angular moment, and energy of a particle around a relativistic source [4]. In general, their formulas depend on the mass, angular momentum, mass- quadrupole, current octupole and mass $2^{4}$-pole moments and on upper order multipoles in the rotation parameter. Including all terms up to order $O(4)$ in the rotation parameter, they found the following equation for the circumferential radius of the corotating ISCO $\left(R_{+}\right)$:

$$
\begin{aligned}
R_{I S C O}^{S \& S} & =6 m\left(1-0.54433 j-0.22619 j^{2}+0.17989 q_{2}\right. \\
& -0.23002 j^{3}+0.26296 j q_{2}-0.05317 q_{3} \\
& -0.29693 j^{4}+0.44546 j^{2} q_{2}-0.06249 q_{2}^{2} \\
& \left.+0.01544 q_{4}-0.11310 j q_{3}\right) .
\end{aligned}
$$

In the previous expression, dimensionless parameters $q_{2}=-M_{2} / m^{3}, q_{3}=-S_{3} / m^{4}$ and $q_{4}=M_{4} / m^{5}$ have been introduced. S\&S adopted the approximation, $q_{4}=$ $\alpha_{4} q_{2}{ }^{2}$ because the numerical calculation of the $2^{4}$-pole term is very hard and difficult. The value of $\alpha_{4}$ lies between 0 and 2, we adopted $\alpha_{4}=1$ as it is generally used [4]. In the case of the Kerr metric, the approximate expression for the location of the co-rotating ISCO up to order $O\left(j^{4}\right)$ is

$$
\begin{aligned}
R_{I S C O}^{K e r r} & =6 M\left(1-0.54433 j-0.04630 j^{2}\right. \\
& \left.-0.02016^{3}-0.01110 j^{4}\right) .
\end{aligned}
$$

In all cases, the location of the counter-rotating ISCO $\left(R_{-}\right)$is obtained simply using the above equation and changing the sign of all the star's rotation multipoles. The obtained results from the direct comparison between the ISCOs are presented in the Fig. [3. 
(a.1)

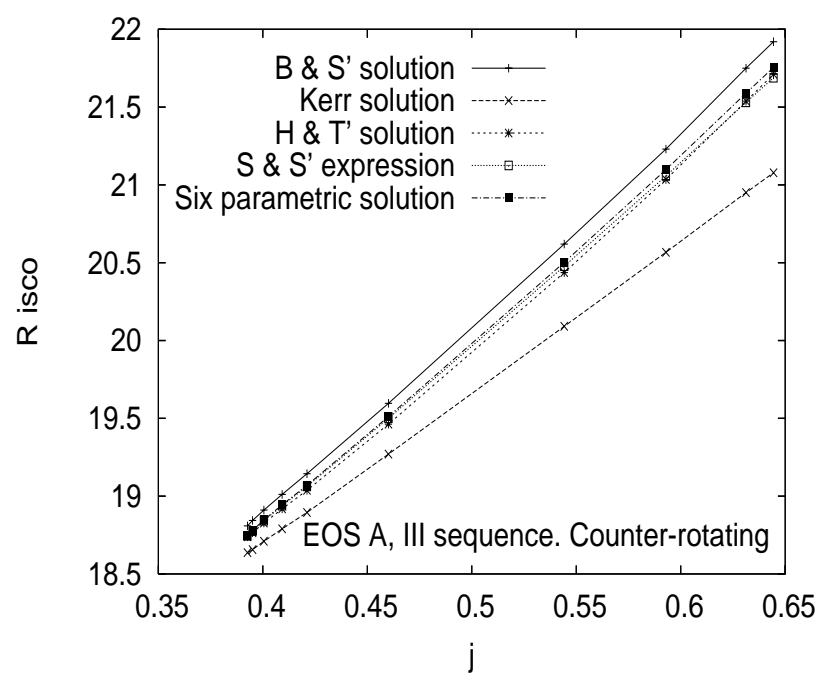

(b.1)

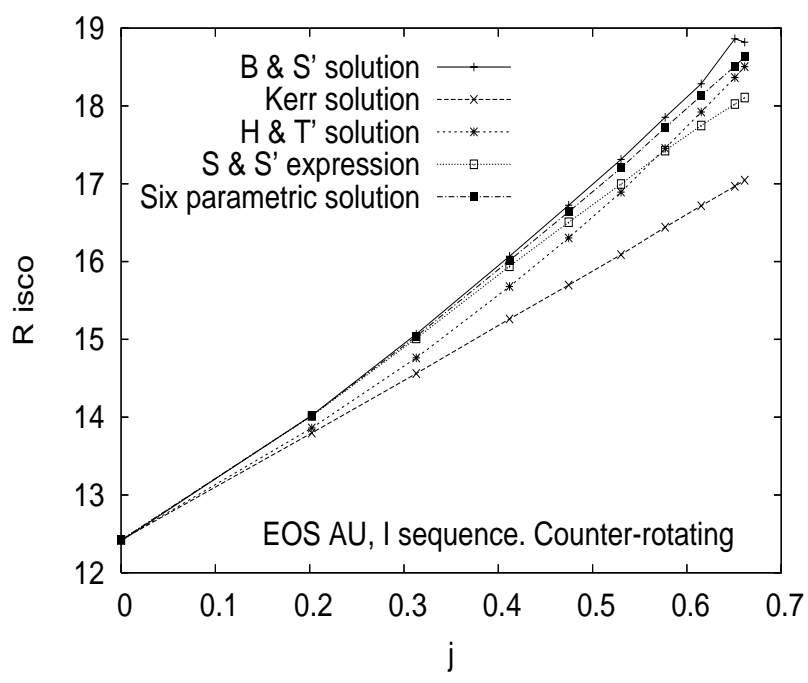

(c.1)

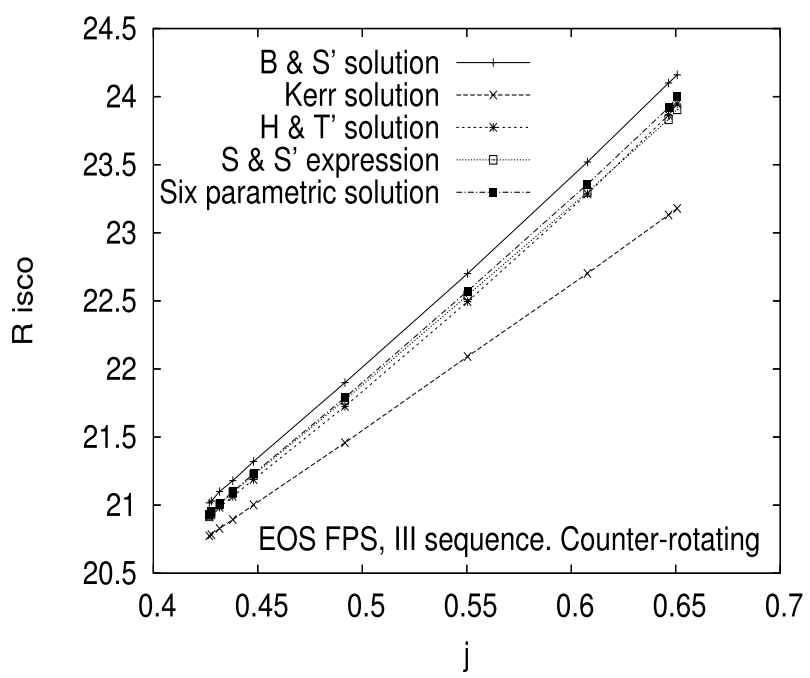

(a.2)

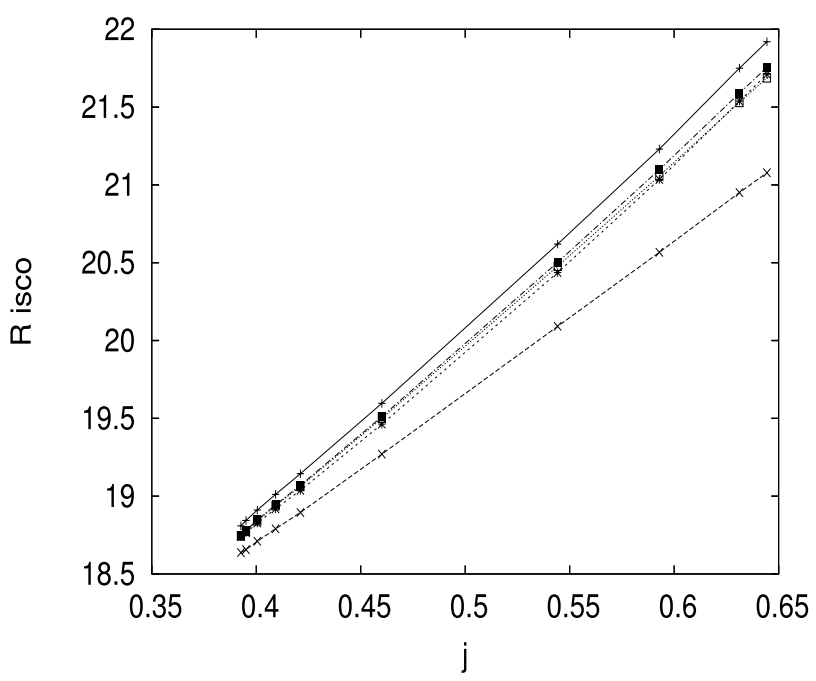

(b.2)

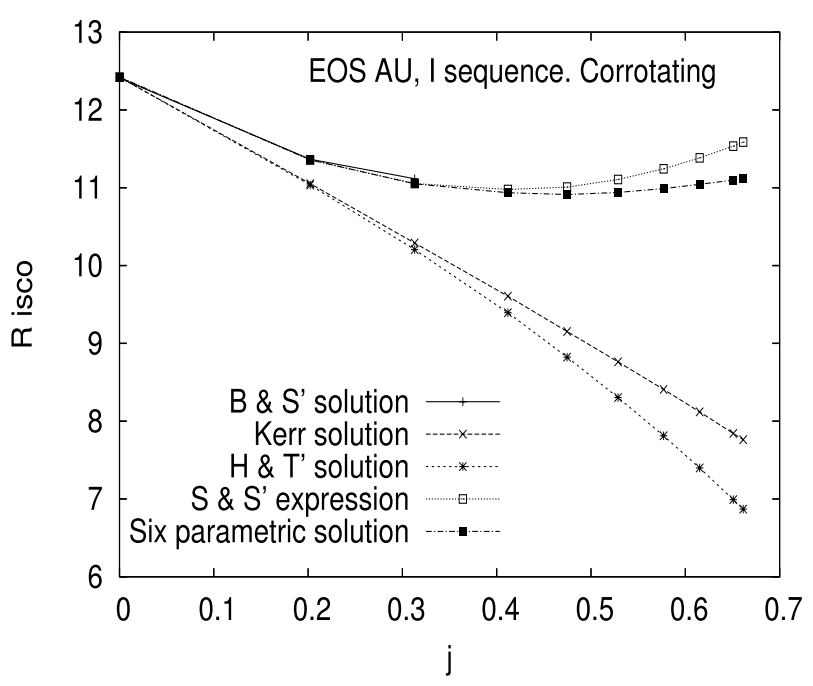

(c.2)

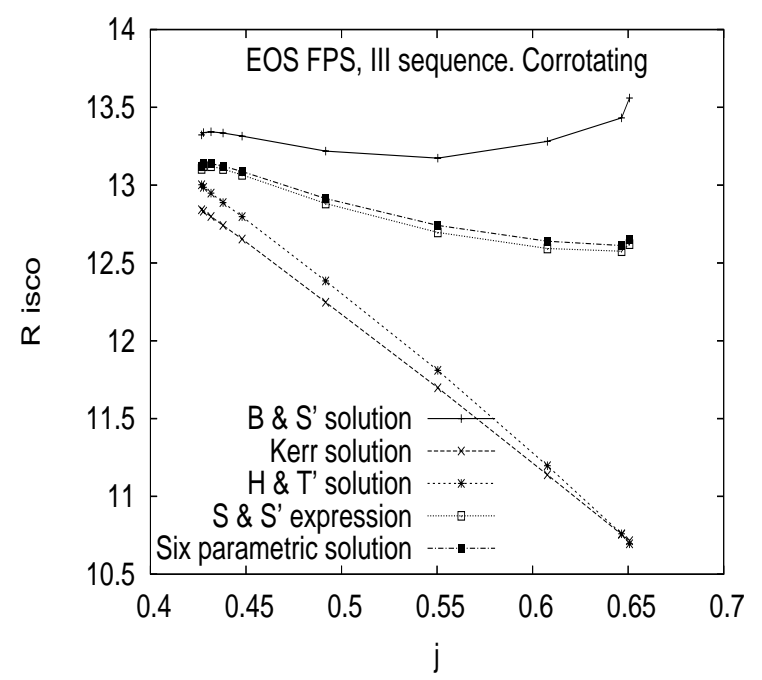


(d.1)

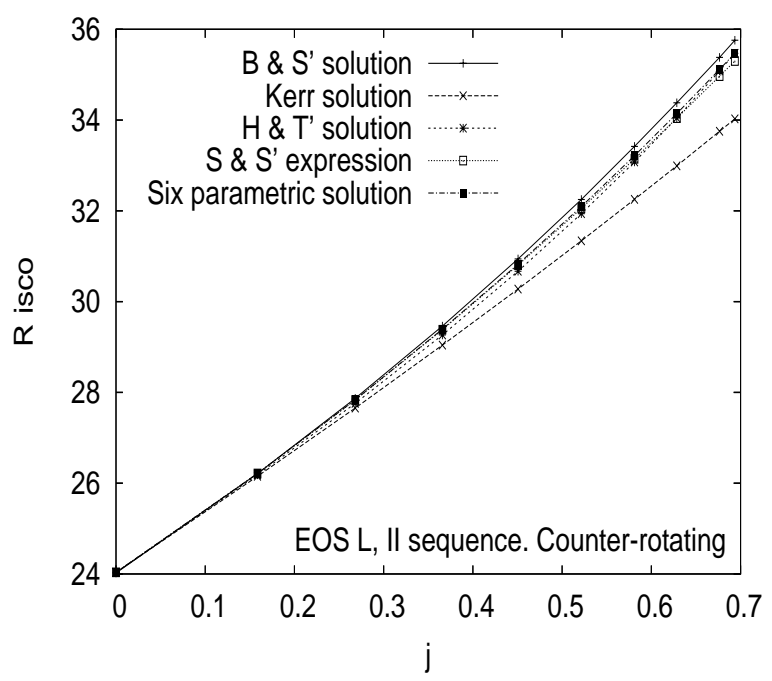

(e.1)

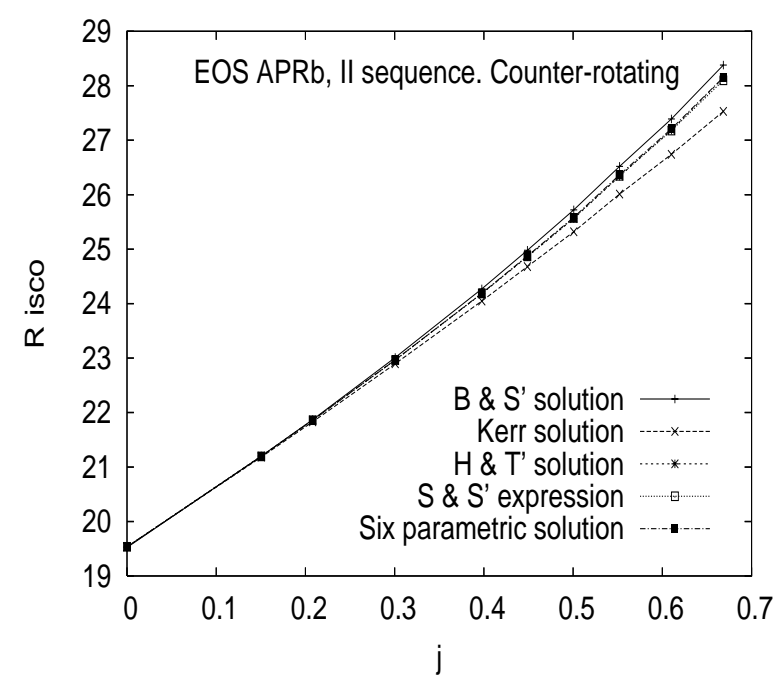

$(\mathrm{d} .2)$

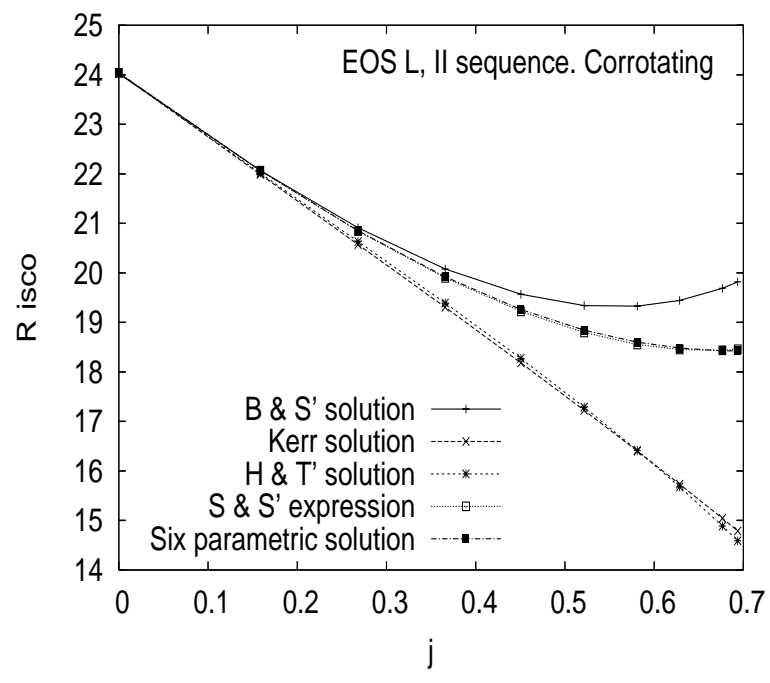

$(\mathrm{e} .2)$

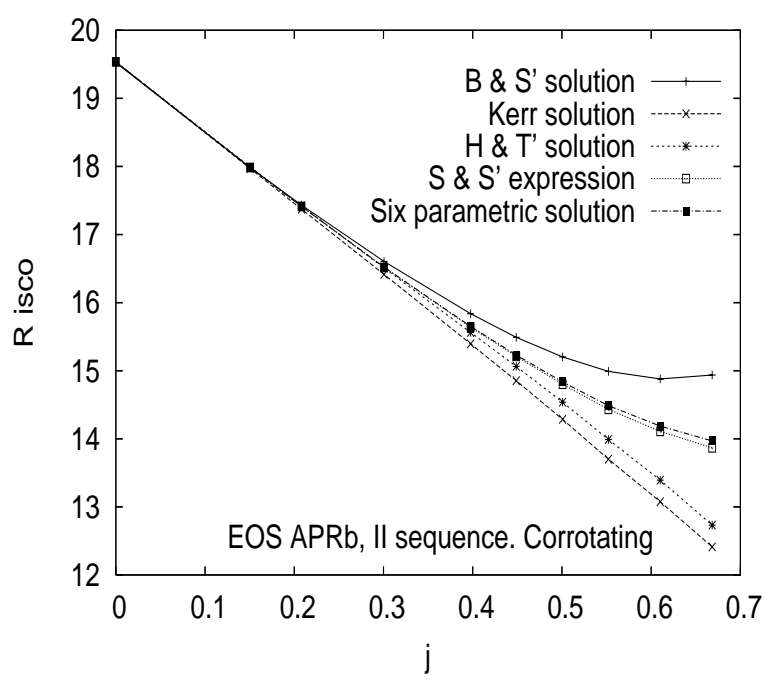

FIG. 3: ISCO for the EOS A sequence with constant rest mass corresponding to a maximum-mass model in the non rotating limit of $1.948 M_{\odot}$ for (a.1) the counter-rotating orbits and (a.2) the co-rotating orbits. ISCO for the EOS AU sequence with constant rest mass corresponding to a nonrotating model of $1.578 M_{\odot}$ for (b.1) the counter-rotating orbits and (b.2) the corotating orbits. ISCO for EOS FPS sequence with constant rest mass corresponding to a maximum-mass model in the non rotating limit of $2.226 M_{\odot}$ for (c.1) the counter-rotating orbits and (c.2) the co-rotating orbits. ISCO for EOS L sequence with constant rest mass corresponding to the maximum-mass model in the non rotating limit $3.232 M_{\odot}$ for (d.1) the counterrotating orbits and (d.2) the co-rotating orbits. ISCO for EOS APRb sequence with constant rest mass corresponding to the maximum-mass model in the non rotating limit $2.672 M_{\odot}$ for (e.1) the counter-rotating o rbits and (e.2) the co-rotating orbits.

We can see that our solution presents a better adjust for the radii of the ISCO in comparison with the standard analytic models used in the literature to describe the exterior gravitational field of a rotating neutron star, e.g. Kerr solution, Hartle \& Thorne' solution (H \& T' solution) and the Shibata \& Sasaki' expressions (S \& S' expression). In average, for all cases the accuracy of $R_{+}$ is better than $2 \%$, and for $R_{-}$better than $1 \%$. The fact that the six parametric solution fits quite well from the non-rotating limit to the rapidly rotations rates, in all 
sequences of mass in the five studied equation of state, implies that it could be an appropriated analytic closed form model for the exterior gravitational field of a neutron star.

\section{CONCLUDING REMARKS}

We present a new stationary axisymmetric six-parameter closed-form analytic solution generalizing to KerrNewmann solution with arbitrary mass-quadrupole moment, octupole current moment and electric charge. The analytic form of its multipolar structure and their electric and magnetic fields, are also presented.

We calculated some properties of the solution, using realistic data of interior numerical solutions, in order to demonstrate that the solution could model the exterior field of a realistic Neutron Star. In the electrovacuum case, we used the magnetic dipolar moment given by Bocquet et al. in [22]; in the vacuum case, we matched it to highly-accurate numerical solutions by Berti \& Stergioulas 1], imposing that the mass, angular momentum, mass-quadrupole and current octupole moment of the numerical and analytic space-times have the same value. We also showed that this six-parameter analytic closedform model is an appropriated model for the exterior field of a slowly or rapidly Neutron Star based on comparisons of the radii of ISCO obtained with a) Berti \& Stergioulas numerical solutions (2004) 1], b) Kerr solution (1963) [2], c) Hartle \& Thorne solution [3], d) an analytic series expansion derived by Shibata \& Sasaki (1998) 4] with our analytic model for all Equations of State given in [1]. However, the solution's accuracy should be tested also outside of the equatorial plane, and through the calculation of other physical observables, which is a task for the future.

The exact solution could be used in future studies of astrophysical plasma, dynamic of geodesics and accretion of particles in the surrounding space-time of the Neutron Star.

\section{Acknowledgments}

J. D. Sanabria-Gómez acknowledges financial support from COLCIENCIAS Colombia. Jorge A. Rueda thanks Universidad Industrial de Santander (Colombia) and Universidad de Los Andes (Venezuela) for the financial support. This work was also supported by Project 5116 (DIEF-Ciencias of the Universidad Industrial de Santander, Colombia). The authors thank to referee for helpful comments.
[1] E. Berti and N. Stergioulas, Mon. Not. Roy. Astron. Soc. 350, 1416 (2004).

[2] R. P. Kerr, Phys. Rev. Lett. 11, 237 (1963).

[3] J. B. Hartle, and K. S. Thorne, Ap. J. 153, 807, (1968)

[4] M. Shibata and M. Sasaki, Phys. Rev. D. 58104011 (1998).

[5] D. C. Backer, S. R. Kulkarni, C. E. Heiles, M. M. Davis, and W. M. Goss, Nature 300, 615 (1982).

[6] Recently, in December of 2005 was discovered the fastestspinning neutron star in history, a $716-\mathrm{Hz}$ eclipsing binary radio pulsar in the globular cluster Terzan 5 using the Green Bank Telelescope by J.W. T. Hessels, S. M. Ransom, I. H. Stairs, P. C. C. Freire, V. M. Kaspi, F. Camilo, Sience March ...

[7] F. M. Curtis, Theory of Neutron Star Magnetospheres (The University Chicago Press 1991).

[8] D. Chakrabarty, E. H. Morgan, M. P. Muno, D. K. Galloway, R. Wijnands, M. van der Klis, C. B. Markwardt, Nature 424, 42 (2003).

[9] E. Berti, F. White, A. Maniopoulou and M. Bruni, Mon. Not. Roy. Astron. Soc. 358, 923 (2005).

[10] Cook G. B., Shapiro D. L. and Teukolsky S. A., Ap. J. 424, 823 (1994).

[11] J. A. Font, H. Dimmelmeier, A. Gupta and N.Stergioulas, Mon. Not. R. Astron. Soc. 325, 1463 (2001).

[12] H. J. Wiebicke, and U. Geppert, Astron. Astrophys. 309, 203 (1996).
[13] C. Kouveliotou et al., Nature 393, 235 (1998). C. Kouveliotou et al., Astrophys. J. 510, L115 (1999). S. Mereghetti and L. Stella, Ap. J. 442, L17 (1995).

[14] R. C. Duncan and C. Thompson, Ap. J. 392, L9 (1992).

[15] H. Komatsu, Y. Eriguchi, I. Hachisu, Mon. Not. Roy. Astron. Soc. 237, 355 (1989).

[16] C. R. Ghezzi, Phys. Rev. D 72, 104017 (2005).

[17] M. E. Gusakov, D. G. Yakovlev and O. Y. Gnedin, Mon. Not. Roy. Astron. Soc. 361, 1415 (2005).

[18] S. Yoshida and Y. Eriguchi, Mon. Not. Roy. Astron. Soc. 347, 575 (2004).

[19] M. Stute and M. Camenzind, Mon. Not. Roy. Astron. Soc. 336, 831 (2002).

[20] V. S. Manko, E. W. Mielke and J. D. Sanabria-Gómez , Phys. Rev. D 61, 081501 (2000).

[21] N. R. Sibgatullin, R. A. Sunyaev, Astronomy Letters 24, 774 (1998).

[22] M. Bocquet, S. Bonazzola, E. Gourgoulhon, J. Novak, Astron. Astrophys. 301, 757 (1995).

[23] Cook G. B., Shapiro D. L. and Teukolsky S. A., Ap. J. 398, 203 (1992).

[24] N. R. Sibgatullin, Oscillations and waves in strong gravitational and electromagnetic fields (Engl. transl.), Springer, Berlin [orig. Russian, 1984, Nauka, Moscow] (1991).

[25] V. S. Manko and N. R. Sibgatullin, Class. Quantum Grav. 10, 1383 (1993). 
[26] N. R. Sibgatullin, Astron. Lett. 28, 83 (2002).

[27] M. A. Abramowicz, G.J.E. Almergren, W. Kluzniak and A.V. Thampan, arXiv:gr-qc/0312070 v1 15 Dec 2003.

[28] J. B. Hartle, ApJ, 150, 1005 (1968).

[29] W. G. Laarakkers and E. Poissson, Astrophys. J. 512, 282 (1999).

[30] V. S. Manko, J. Martin, E. Ruiz, N. R. Sibgatullin, M. N. Zaripov, Phys. Rev. D, 49, 5144 (1994).

[31] V. R. Panaripande, Nucl. Phys. A 174, 641 (1971).

[32] R. B. Wiringa, V. Fiks and A. Fabrocini, Phys. Rev. C 38, 1010 (1988).

[33] C. P. Lorenz, D. G. Ravenhall and C. J. Pethcik, Phys. Rev. Lett. 70, 379 (1993).

[34] A. Tomimatsu and H. Sato, Phys. Rev. Lett. 29, 1344 (1972).

[35] V. R. Panaripande and R. A: Smith, Nucl. Phys. A 237, 507 (1975).

[36] A. Akmal, V. R. Pandharipande, D. G. Ravenhall, Phys. Rev. C 58, 1804. (1998).

[37] L. Lichtenstein, 1933, Gleichgewichisfiguren rotierender Flssigkeiten (Berlin: Springer)

[38] L. Lindblom, Phil. Trans. R. Soc. A. 340, 353 (1992).

[39] A. Papapetrou, Ann. Phys. 12, 309 (1953).
[40] F. J. Ernst, Phys. Rev. 168, 1415 (1968).

[41] L. A. Pachón and J. D. Sanabria-Gómez, Class. Quantum Grav 23, 3251 (2006).

[42] The form of the potential $e(z)$ was supplied by the undergraduate students Cesar A. Valenzuela and Fabio D. Lora; they calculated it under the directions of J. D. S.-G.

[43] C. Hoenselaers and Z. Perjés, Class. Quantum Grav. 7, 1819 (1990).

[44] N. Breton, V. S. Manko, and J. A. Aguilar-Sanchez, Classical Quantum Gravity 16, 3725 (1999).

[45] L. Herrera, G. A. González, L. A. Pachón and J. A. Rueda, Class. Quantum Grav. 232395 (2006).

[46] W. B. Bonnor, Z. Phys. 190, 444 (1966).

[47] Robert M. Wald, General Relativity, The University of Chicago Press. (1984)

[48] W. Kinnersley, J. Math. Phys. 18, 1529 (1977).

[49] V. C. A. Ferraro, Astrophys. J. 119, 407 (1954).

[50] A. Tomimatsu, Prog. Theor. Phys. 70, 385 (1983).

[51] V. S. Manko, E. Ruiz and J. D. Sanabria-Gómez, Class. Quantum Grav. 173881 (2000).

[52] J. M. Bardeen, Ap. J. 161103 (1970). 